\title{
Automatic control system of vacuum nano coating based on AVR single chip microcomputer
}

\author{
Jinwen Li, Jie Zhang*, \\ Department of Electrical and Electronic Engineering, Chengde Petroleum College, \\ Chengde, 067000 \\ China

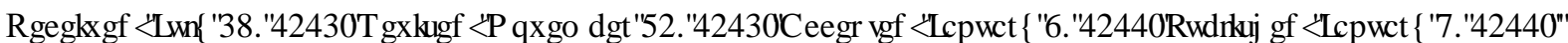

\begin{abstract}
Aiming at the problems of long response time and poor anti-interference ability of traditional vacuum nano-coating automatic control system, a design of vacuum nano-coating automatic control system based on AVR single-chip microcomputer is proposed. The use of ATmega128L microcontroller and Harvard architecture improves the parallel processing efficiency of the microcontroller. Select the transient voltage suppression diode to protect the power supply of the single-chip microcomputer, optimize the communication circuit, connect an external encryptor to realize the data encryption function, increase the filtering program and optimize the binary code processing function. Using PID (Packet Identifier) control algorithm, the design of nano-coating vacuum automatic control system based on AVR single-chip microcomputer is realized. Compared with the traditional system, when the simulation model and system parameters of the control system constructed in the environment change, the proposed system can be stabilized within 15 seconds, and can be stabilized for 13 seconds after the interference signal is added. The response time of the system is longer. Shorter, stronger anti-interference ability, more suitable for automatic control of vacuum nano-coating.
\end{abstract}

Keywords-AVR Single Chip Microcomputer, Coatings, Characterisation, Design.

\section{INTRODUCTION}

$\mathrm{V}$ ACUUM coater mainly refers to a kind of coating which needs to be carried out under high vacuum, including many kinds, such as vacuum ion evaporation, magnetron sputtering, MBE (Molecular beam epitaxy) molecular beam epitaxy, PLD (programmable logic device) laser sputtering deposition, etc. The main idea is divided into evaporation and sputtering. The substrate to be coated is called substrate, and the material to be coated is called target. The substrate and target are in the same vacuum cavity. Evaporation coating is generally used to heat the target so that the surface components are evaporated in the form of atomic clusters or ions. Moreover, the film is deposited on the substrate surface and formed by the film forming process of scattered-island structure-stray structure-layered growth. For sputtering type coating, it can be simply understood that the target is bombarded with electrons or high-energy laser, and the surface components are sputtered out in the form of atomic clusters or ions, and finally deposited on the substrate surface, going through the film-forming process, and finally forming the film. The automatic control system should be able to realize the following services: It can record the measured values of all controlled quantities at any time in the process, for the time coordinate curve of analog quantity equivalent to the given measured value and for the truth table of logic quantity equivalent to the given value to time. It is required to be easy to prepare and read, and be able to conduct self-action statistical analysis; Real-time control and adjustment of running equipment can be conducted through HMI (Human Machine Interface); A given process file can be selected from the process menu for automatic execution; A new process can be prepared and filed for execution; Each power supply is provided with current, output current and output voltage; As well as currently executed process menu name, current process stage, execution time and remaining time; The current operation curve and historical curve can be displayed during operation; The analog quantity is over limit, and the protection signal can be automatically switched and displayed, the alarm sound can be started, and the manual release can be accepted; The relevant technical documents can be consulted. The important parameters of each operation process are archived as historical records, including real-time simulation, switching value and additional explanation in the later stage; Each operation can generate an example process file, which can be re executed once after being transferred in; The control system has the functions 
of data transmission, data management, data backup, data statistics summary, report printing, etc., so as to facilitate the process analysis and experimental improvement on the basis of calculation.

Because the traditional automatic control system of vacuum nano coating does not take the data processing efficiency as the research focus in the design process, which leads to long response time and poor anti-interference ability of the system. In order to solve this problem, a design scheme of automatic control system of vacuum nano coating based on AVR microcontroller is proposed. AVR single chip microcomputer is a high speed 8-bit single chip microcomputer of RISC (Reduced Instruction Set Computer) reduced instruction set with enhanced built-in flash developed by ATMEL company in 1997 [1]-[3]. It can be widely used in computer external equipment, industrial real-time control, instruments and meters, communication equipment, household appliances and other fields. AVR microcontroller is RISC microcontroller introduced by ATMEL company in 1997. RISC is relative to CISC. RISC is not simply to reduce the number of instructions, but to improve the operation speed by making the structure of the computer more simple and reasonable. RISC preferentially selects simple instructions with the highest frequency to avoid complex instructions, and fixes the instruction width to reduce the type of instruction format and addressing mode, so as to shorten the instruction cycle and improve the operation speed. Because AVR adopts RISC structure, AVR Series MCU (Microcontroller Unit) has the high-speed processing capacity of $1 \mathrm{MIPS} / \mathrm{MHz}$. Because of the low level of technology and design, high power consumption and poor anti-interference performance, the early single-chip microcomputer adopted a safe scheme: Using a high frequency division coefficient to divide the clock frequency, which makes the instruction cycle long and the execution speed slow. In the future, although the CMOS (Complementary Metal Oxide Semiconductor) single chip microcomputer adopts measures such as increasing the clock frequency and reducing the division coefficient, this state has not been completely changed. Although there are some single chip computers reducing instruction set, they still follow the practice of clock frequency division. The introduction of AVR single-chip computer completely breaks the old design pattern, abolishes the machine cycle and abandons the complex instruction computer's practice of pursuing complete instruction. It adopts the simplified instruction set, takes the word as the instruction length unit, and arranges the operation number and operation code with rich content in one word, with short index cycle and prefetchable instruction, realizing flow operation, so it can execute the instruction at high speed. Of course, this speed jump is backed by high reliability [4]-[6].

Therefore, the system can fully solve the problems existing in the traditional system, reduce the system response time and improve the anti-interference ability of the system. It has a certain reference significance for the performance optimization of the vacuum coating machine, can promote the further development of the industrial processing field, and has a very prominent contribution to the enrichment and development of the research in the field of automatic control.

\section{DESIGN Of AUtomatic CONTROL SyStem OF VACUUM NANO COATING BASED ON AVR Single CHIP MICROCOMPUTER}

\section{A. Hardware Design of Automatic Control System of Vacuum Nano Coating Based on AVR Single Chip Microcomputer}

Selection of AVR singlechip. ATmega128L single chip computer of ATMEL company is selected. ATmega128L is an 8-bit MCU based on AVR core, RISC structure and low power CMOS. In order to improve the parallel processing efficiency of MCU, AVR MCU adopts Harvard structure of program memory and data memory with different storage space and access bus. The ALU (Arithmetic and Logic Unit) accesses the program memory by using the single-stage pipeline operation mode. While executing the current instruction, it also completes the operation of taking one instruction to be executed from the program memory. Therefore, an instruction only needs one clock cycle [7]-[9]. Because one instruction is executed in one clock cycle, ATmega128L can achieve performance close to 1 MIPS/MHz, which can alleviate the contradiction between power consumption and processing speed of the system. The core structure of AVR MCU is shown in the Fig. 1. 


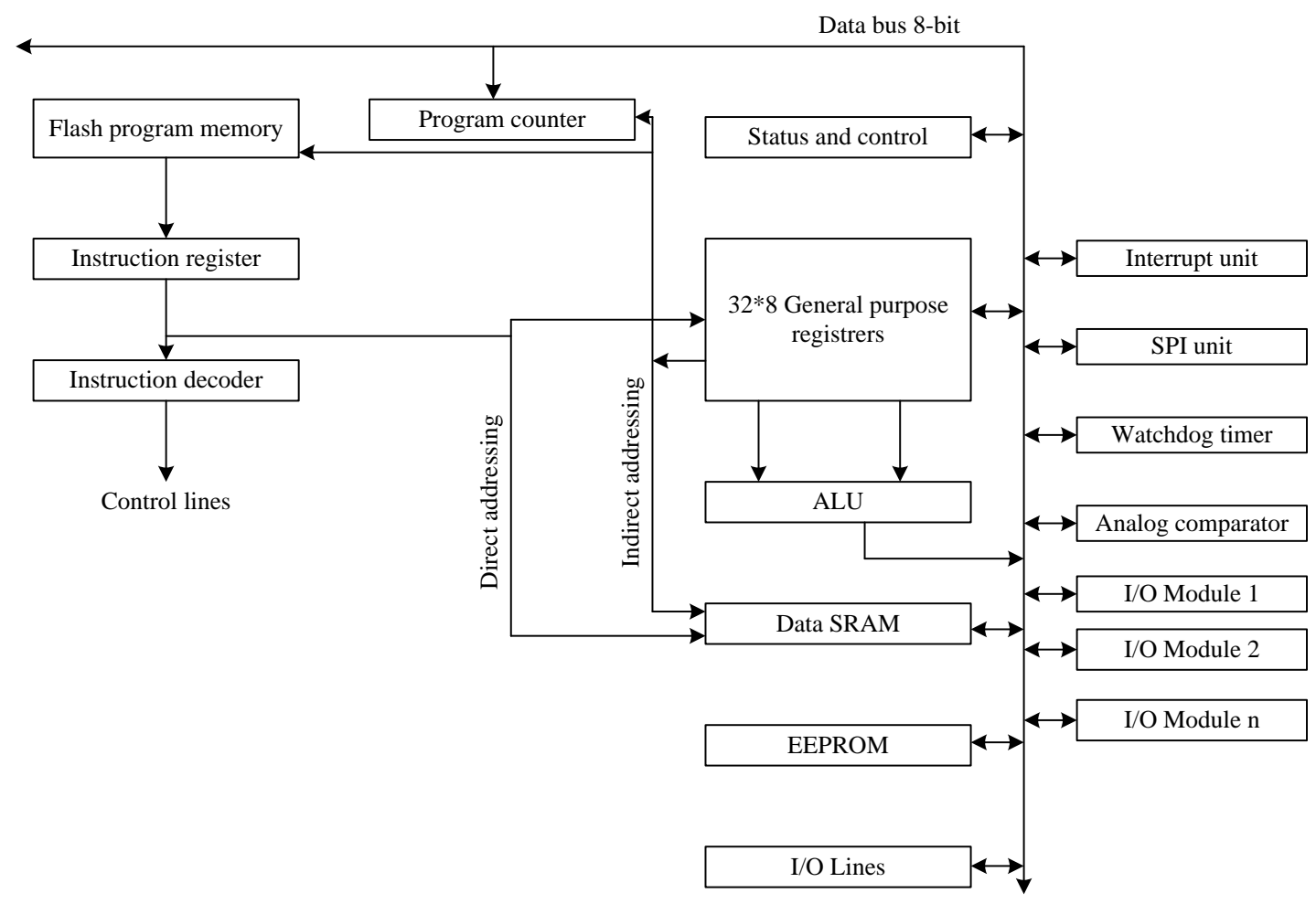

Fig. 1 Core structure of AVR single chip microcomputer

In the AVR kernel, the "fast access register group" is composed of 32 general 8-bit working registers that only need one clock cycle for access operations. In these registers, only one clock cycle is needed to complete a complete ALU operation. This means that the three processes of fetching two operands from the register, performing operation and writing the result back to the register are completed in one clock cycle, which is called "fast access". The interrupt control of AVR consists of the interrupt control register in the $\mathrm{I} / \mathrm{O}$ register space and the global interrupt allow bit in the status register. Each interrupt has an interrupt vector corresponding to it. All interrupt vectors form interrupt vector table, which is located at the front of program memory space. The smaller the interrupt vector address is, the higher the priority is. The $\mathrm{I} / \mathrm{O}$ space is a continuous $64 \mathrm{I} / \mathrm{O}$ register space, corresponding to the control and data register address of each peripheral function of MCU, and the mapping address in the data storage space is 0020-005 F. The characteristics of ATmega128L can well meet the design requirements of the system for the control chip, especially its rich internal and external interrupt sources can control the intelligent power supply and distribution of the system in time, and the two enhanced programmable serial USART (Universal Synchronous/Asynchronous Receiver/Transmitter) interfaces can respectively communicate with the encryption machine and the upper computer for data transmission. Design of power supply and reset circuit of ATmega128L as: Due to the particularity of the application field of the system, it needs to withstand the electromagnetic impact of additional $200 \mathrm{~V}, 10$ $\mu \mathrm{m}$ pulse interference for $30 \mathrm{~s}$. In order for the single chip computer to work normally, it is necessary to add voltage stabilizing and filtering circuit at the power supply end [10].
The transient voltage suppression diode SY6039 is selected to protect the power supply end of the single chip microcomputer, as shown in the Fig. 2.

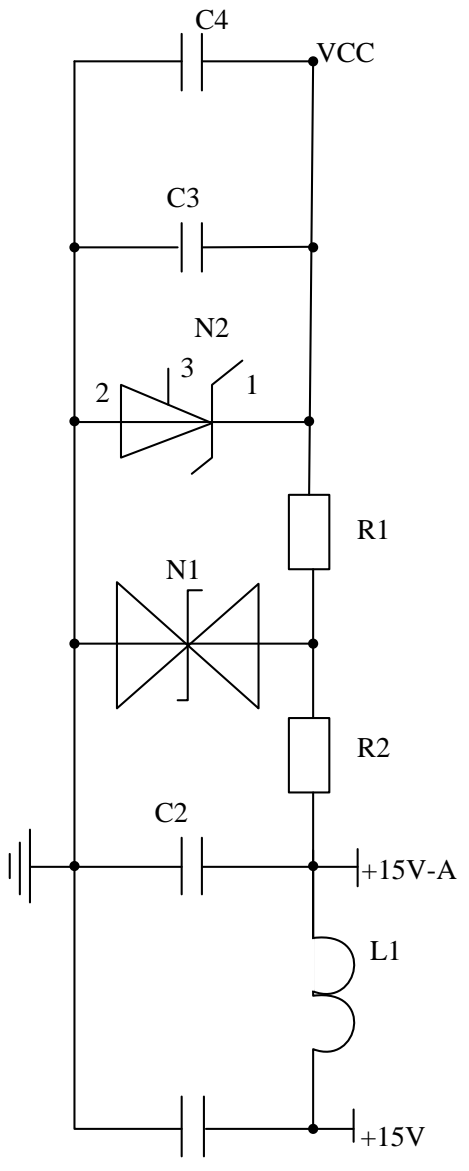


Fig. 2 Power protection filter circuit

When impacted by external high voltage, the transient voltage suppresses diode $\mathrm{N} 1$ and clamps the voltage value to 15 $\mathrm{V}$. The voltage reference $\mathrm{N} 2$ of the back end is ADR550ART of AD company. Its input voltage is $15 \mathrm{~V}$. After the chip starts to vibrate normally, $5 \mathrm{~V}$ voltage can be generated for the use of the single chip microcomputer. In order to ensure the stability of power signal, RC (Release Candidate) network is used for filtering. In order to ensure that the oscillator is stable when the single chip microcomputer starts to work normally, the CPU has a delay from power on to normal operation, and the watchdog oscillator is used as the timer of the start delay [11]. The external crystal oscillator is selected as the clock source of the system. A quartz crystal oscillator is connected at XTAL1 and XTAL2 (XTAL = External Crystal Oscillator) pins of ATmega128L to form the clock source. The wake-up delay time of $4.1 \mathrm{~ms}$ is selected by fuse bits CKSEL0 and SUT1 - 0 .

Design of communication circuit. In order to enable the upper computer to monitor different access devices, the Ethernet transmission PC monitoring mode based on MODBUS is adopted. One of the two serial interfaces USART is used to communicate with the upper computer. The USART of ATmega128L supports 5, 6, 7, 8 and 9 data bits, 1 or 2 stop bits serial data frame structure, which can well support Modbus protocol. The connection block diagram of communication part is in Fig. 3.

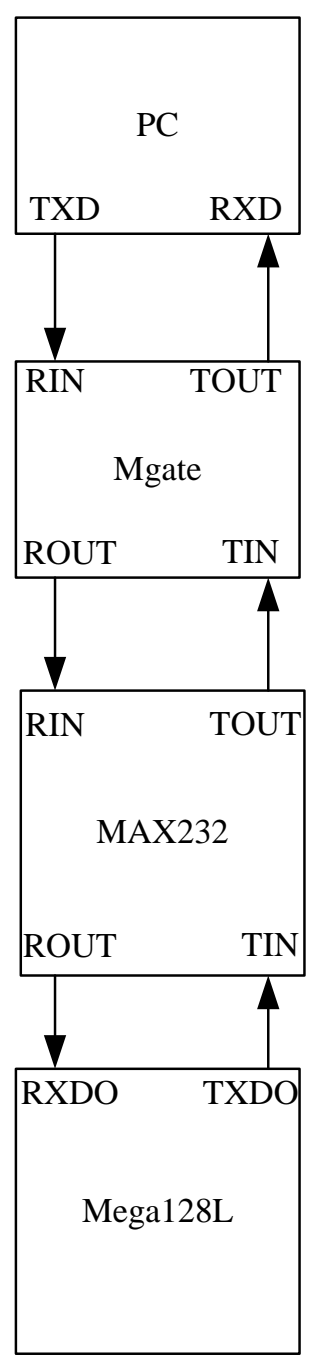

Fig. 3 Communication connection block diagram

When the upper computer communicates with the single-chip microcomputer, the computer uses positive and negative voltage to represent the logic state, the state of the effective negative level is logic 1 , and the state of the effective positive level is logic 0; While the TTL (Transistor Transistor Logic) level used by the single-chip microcomputer uses high and low level to represent the two logic states of 1 and 0 , so the level conversion interface chip of the level logic relationship transformation must be added to the communication between the two. In this paper, MAX232AMJE, the 232 bus transceiver of MAXIM company, is selected to convert TTL and RS232 into logic level. In order to enable the upper computer to control multiple slave devices with different interfaces, the MGate MB3170T gateway module of Mosa company is used to convert the TCP (Transmission Control Protocol) and RTU (Remote Terminal Unit) protocols.

Encryptor design. Due to the DES (Data Encryption Standard) algorithm realized by SCM (Software Configuration Management) software has a large amount of calculation, which will consume too much processor resources, the way of external encryptor is chosen to cooperate with SCM to realize the data encryption function. The encryptor is used for real-time 
encryption of telemetry information, and its main functions include: Real-time encryption / decryption of PCM (Pulse Code Modulation) unframed information; Ground encryption/decryption inspection function; Manual destruction of key data; Emergency destruction of password and key data. Its performance and technical indicators are as follows: Encryption mode is one encryption per frame; Encryption and decryption rate is more than $10 \mathrm{Mbps}$; encryption and decryption delay are less than $1 \mathrm{~ms}$. The cipher algorithm program is solidified in the non-volatile memory. After the data acquisition system is powered on, the algorithm FPGA (Field Programmable Gate Array) is automatically constructed and configured, and the self-check of FPGA algorithm is started. After the algorithm self-check is successful, it waits for the encryption and decryption call of the data processing main system [12].

The second USART interface of ATmega128L is used to communicate between MCU and data encryptor. When encrypting data, under the control of single chip microcomputer, FPGA receives the data of single chip microcomputer through the interface designed by FPGA, transmits it to the encryption module and sends it back to the interface after encrypting. When it sends the request reading signal to the single chip microcomputer, the single chip microcomputer responds to complete the data encryption [13]. The schematic diagram is shown in the Fig. 4. FPGA is treated as a common interface chip in the figure. FPGA can be selected and read-write operation can be carried out through chip selection nCS, read-write signal RD and nWR. When FPGA outputs data to MCU, the interrupt signal outputs INT trigger level. After MCU responds, it can read the interrupt details in FPGA state register and make corresponding interrupt processing.

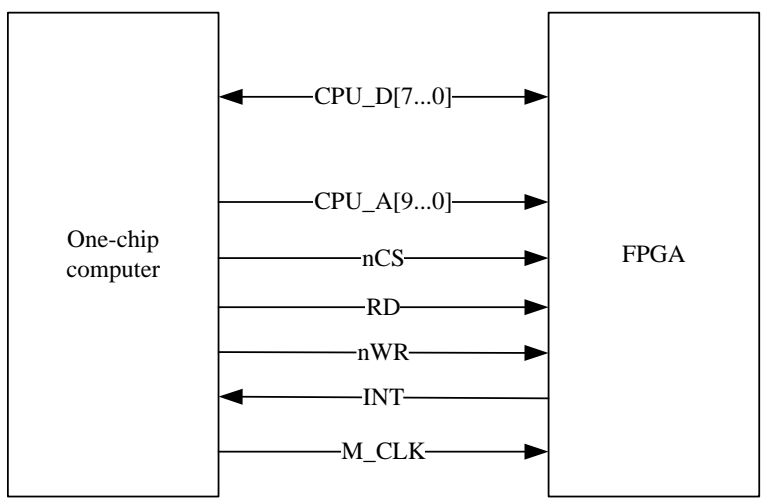

Fig. 4 One-chip computer and FPGA node principle block diagram

Interface module design: the interface module is mainly used for data transmission with single-chip microcomputer, and data storage and reading in specified register or buffer. The interface module provides a clock to FPGA and reads and writes data to FPGA through address, data bus and read-write signal, sends and receives buffer memory, control register, and address register, etc. The control register is a write only register, including setting baud rate, system reset, start sending, reply sending and receive interrupt.
The higher the clock frequency is, the higher the speed of processing data signal is, but the power consumption of the chip will also be increased accordingly. $20 \mathrm{MHz}$ clock is selected. With the width of MCU data bus, the interface adopts 8-bit data total CPU_D [7...0]. The width of the address bus mainly depends on the size of the data buffer needed. Considering that the single-chip microcomputer also needs to implement some control on the FPGA, there is a certain address left in the FPGA to arrange the control register and the status register, so the address bus should be widened a little. 10 is selected as the width of the address bus CPU_A [9...0].

Encryption module design: In the encryption algorithm module data DATA_DES, a set of key transformation round function and data encryption key operation round function are realized by hardware. The hardware structure is called 16 times repeatedly to realize a DES encryption operation. The schematic diagram is shown in the Fig. 5:

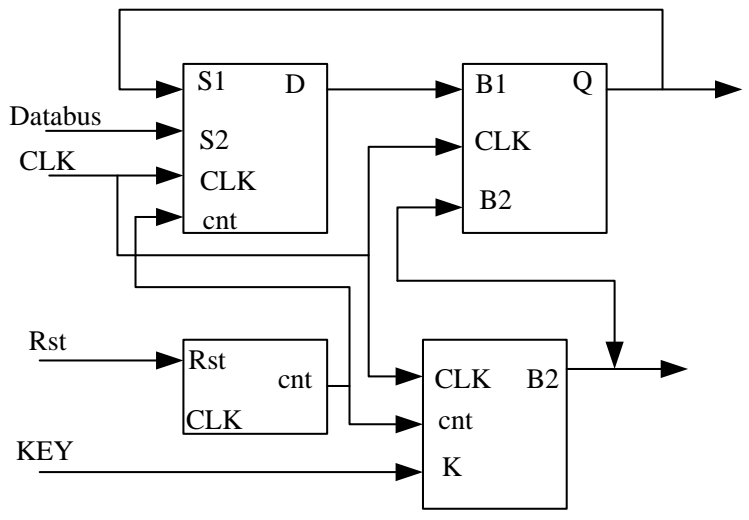

Fig. 5 Schematic diagram of encryption function

Under the control of the wheel counter, the plaintext data Databus and the KEY are sent to the B1 end of the round function after the data transformation processing when the CLK arrives. After the first round of transformation, the data is locked by the register. When the next CLK arrives, the data is sent to the round function inlet together with the corresponding wheel key again. After 16 rounds of cycling, the final result is output. The round counter controls the whole encryption process and is the core of the whole encryption process. The multiplex data selector selects whether the data entering the round function is the original data or the encrypted process round data.

\section{B. Software Design of Automatic Control System for Vacuum Nano Coating}

Modular design of interface software. The software design of the automatic control system for vacuum nano coating includes interface programming and process programming. For the interface program, not only the function of each interface circuit can be realized, but also the standard function form should be written to realize the efficient and robust modular software design. It is not only convenient for the development and design of later process program, but also used for other development [14]. Based on the working principle of the interface software in the early stage, the modular interface software is redesigned and 
optimized according to the $\mathrm{C}$ programming specification, and the experiment debugging is successful. In addition, the filtering program is added from the perspective of software anti-interference. The specific standardized design of interface software is as follows: Before the normal operation of the controller, the initialization work is carried out first, and the successful operation of the initialization program is the basis for the successful operation of the subsequent parts of the software. The initialization program of interface software includes: The initialization of port of single chip microcomputer PIC_Ports_Init() (PIC = single-chip microcomputer), the initialization of digital input and output DI_GateOff_Init (), DO_Latch_Init(), the initialization of analog input and output: AD_Convert_Init(), DA_Convert_Init(),serial communication initialization: SerialComm_Init (), the initialization of FPGA processing encoder signal: FPGA_Encoder_Init(). In order to achieve the standardized compilation and modular design of all the interface programs (including the input and output with clear functions), and facilitate the later process software development, the modular design and debugging of all the interface software are successful. In addition, software filtering is added to the $\mathrm{AD}$ analog quantity collection part from the perspective of software anti-interference to improve the accuracy of AD sampling.

FPGA processing and coding design. According to the actual experimental needs, the software design module of FPGA processing encoder output binary code form is added, absolute value encoder software module becomes optional mode of different output signal form, improving the software processing function. The specific differences between gray code and binary code are as follows: Gray code is a kind of non-weighted code, belonging to absolute coding mode, between any two adjacent codes. When converting, only one bit is different, and there is only one bit difference between the highest bit and the lowest bit, so it is a kind of one-step self-complement code with reflection and loop characteristics. When gray code is used to represent data, whenever data is updated, only one digit value will be changed, and there is no sequence. Binary code is natural binary code. The corresponding relationship between binary code and gray code is shown in Table I:

Table I Correspondence between binary code and gray code

\begin{tabular}{ccc}
\hline Decimal Code & Binary Code & Gray Code \\
\hline 0 & 0000 & 0000 \\
1 & 0001 & 0001 \\
2 & 0010 & 0011 \\
3 & 0011 & 0010 \\
4 & 0100 & 0110 \\
5 & 0101 & 0111 \\
6 & 0110 & 0101 \\
7 & 0111 & 0100 \\
8 & 1000 & 1100 \\
9 & 1001 & 1101 \\
10 & 1010 & 1111 \\
11 & 1011 & 1110 \\
12 & 1100 & 1010 \\
\hline
\end{tabular}




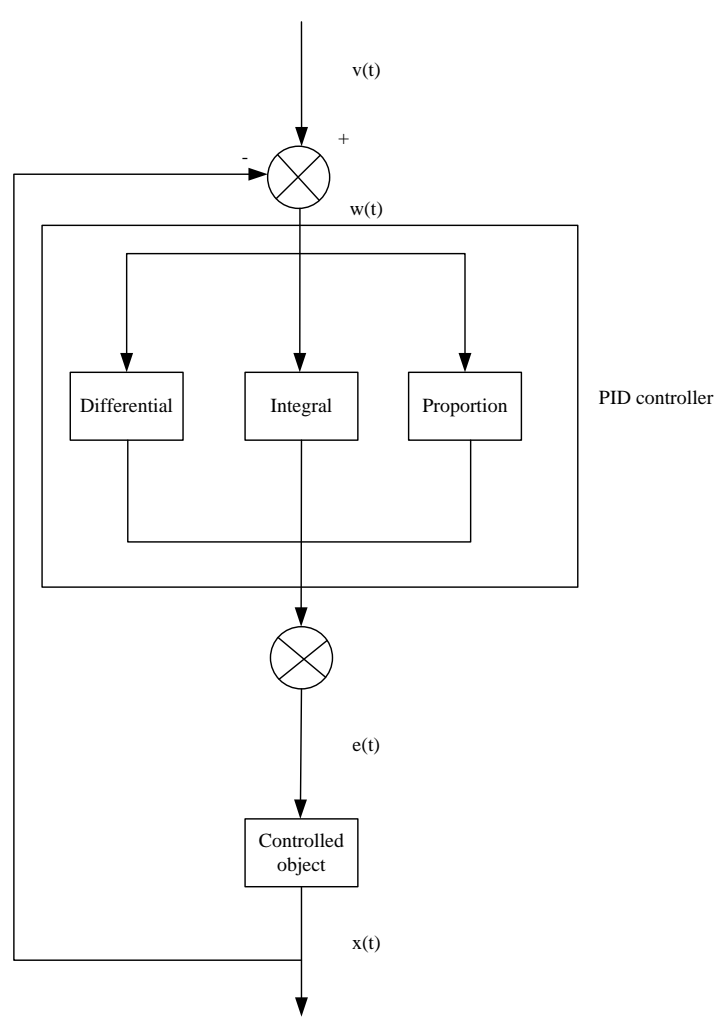

Fig. 7 PID controller structure diagram

As shown in the figure, the deviation of PID controller is mainly affected by two parameters, namely, the given value $v(t)$ and the output value $x(t)$.

$w(t)=v(t)-x(t)$

The control law is as follows:

$e(t)=I_{n} w(t)+\frac{1}{T_{u}} \int{ }_{0}^{t} w(t)+T_{b} \frac{b w(t)}{b t}$

where $I_{n}$ is the proportional coefficient, $T_{u}$ is the integral time constant and $T_{b}$ is the differential time constant.

In short, if there is a deviation in the proportion link, the controller will start to act, so as to reduce the deviation as much as possible. The core function of the integration link is to reduce the steady-state error. The magnitude of the integration function is usually affected by the integration time constant. If $T$ is relatively small, its integration function is relatively large. The differential link is mainly to reflect the change rate of the deviation signal, and can add the correction signal to adjust the dynamic characteristics of the system, thus greatly reducing the adjustment time. The application of PID control algorithm needs to rely on accurate mathematical model, but the actual system is often nonlinear and time invariant, so it is difficult to build up accurate mathematical model. The performance of traditional PID controller is often poor due to poor parameter setting [15]. With the development and deepening of control technology in theoretical research, there are many improved PID controllers, whose control effect has been greatly improved compared with conventional PID control. The final output expression of PID controller is obtained as follows:
$E_{n}(i)=I_{n} w(i)+I_{U} T_{A} \sum_{u=0}^{i} w(u)+\frac{I_{B}}{T_{A}} \Delta w(i)$

where $T_{A}$ is the sampling time; $i$ is the number of samples; $w(i)$ is the error; $\Delta w(i)$ is the rate of change of the error; $I_{n}, I_{U}, I_{B}$ is the proportional, integral and differential gain respectively.

Through the above analysis of the design of expert fuzzy PID control algorithm, the control system performance can be optimized and kept in the best state. So far, the design of the automatic control system of vacuum nano coating based on AVR single chip microcomputer has been completed, and the control experiment has been designed to test the performance of the system.

\section{EXPERIMENTAL RESEARCH}

\section{A. Experiment Process}

In order to verify the performance of the vacuum nano coating automatic control system based on AVR single chip microcomputer, the anti-interference comparison test and execution time comparison test are carried out between the system designed in this paper and the traditional automatic control system. This experiment uses MATLAB mathematical software as the simulation experimental environment platform.

MATLAB is commercial mathematical software produced by MathWorks company in the United States. It is used for algorithm development, data visualization, data analysis and high-level technical calculation language and interactive environment of numerical calculation, mainly including MATLAB and Simulink. MATLAB is a combination of matrix and laboratory, which means matrix factory. It is a high-tech computing environment mainly facing scientific computing, visualization and interactive programming released by MathWorks company of the United States. It integrates many powerful functions such as numerical analysis, matrix calculation, scientific data visualization and modeling and Simulation of nonlinear dynamic system into an easy-to-use window environment, providing a comprehensive solution for scientific research, engineering design and many scientific fields where effective numerical calculation is necessary, and to a large extent, it gets rid of the editing mode of the traditional non-interactive programming language and represents the advanced level of the international scientific computing software. Simulink is entered in the command, and a window of Simulink Library Browser will appear on the desktop. In this window, the names of various modules classified by function are listed as follows: 


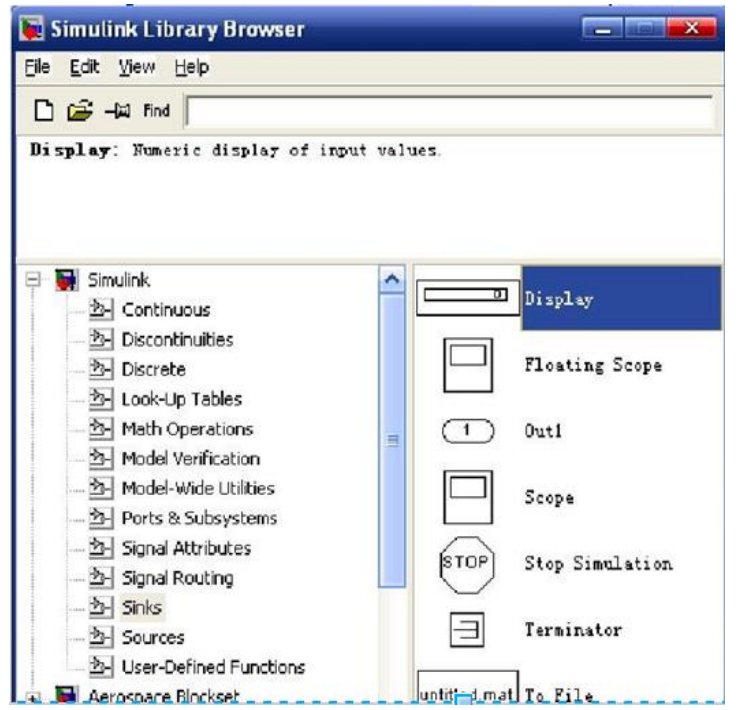

Fig. 8 Simulink toolbox

Due to the high requirements of MATLAB software for the experimental platform, computer of model T58-V is selected as the experimental platform, which uses the ninth generation of intelligent Intel Core $i 7$ processor, has six core and 12 thread CPU, independent 4 GB memory capacity, which can meet the smooth progress of the experiment. In the experimental process, the research and development data of a large nano vacuum coating manufacturer are taken as the experimental sample data. After the data samples are collected, the experimental data need to be cleaned and restored to improve the authenticity and scientific nature of the simulation experiment results. After the preparation of the experiment, the designed system is compared with the conventional automatic control system, and the results are analyzed and compared to draw a conclusion.

\section{B. Experimental Results}

By constructing the simulation model of the control system in the environment, the change curves of the step response of the conventional automatic control system and the designed system are obtained as follows. When the parameters of the system change, the step response curve of the system also changes. In order to test its robustness, an interference signal with amplitude of 0.1 is added in $40 \mathrm{~s}$, and the simulation effect diagram of conventional control and designed control system is obtained, as shown in the following Fig. 9.

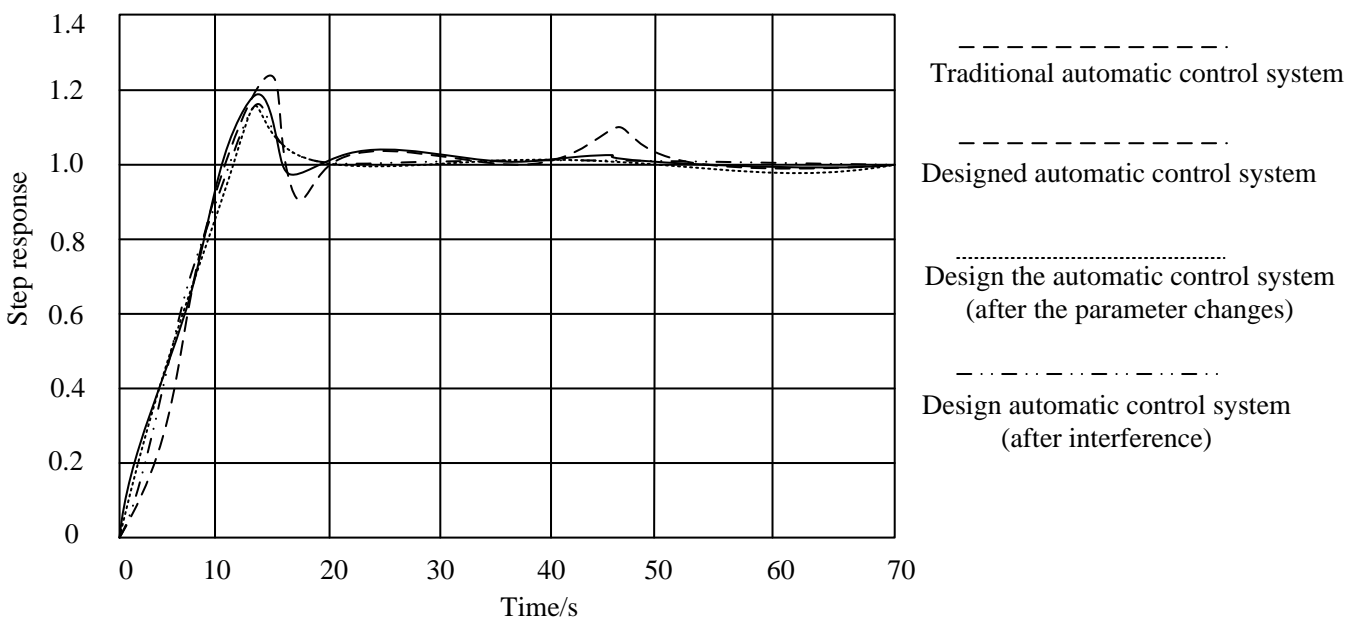

Fig. 9 Current step response curve

By analyzing the data in Fig. 9, it can be seen that the step response of the traditional automatic control system is always fluctuating between 0-53 s and tends to be stable after $53 \mathrm{~s}$. Compared with the system, the step response of the system designed in this paper can approach the stable value faster. When the simulation model and system parameters of the control system constructed in the environment change, the system designed in this paper can tend to be stable in 15 seconds, and can be stable in 13 seconds after the interference signal is added. However, the traditional control system has a longer time and larger amplitude of step response. It can be proved that the dynamic performance and anti-interference ability of the vacuum nano coating automatic control system based on AVR microcontroller have been greatly improved.

On this basis, the control execution time of the automatic control system simulation model constructed in this paper is compared with the traditional two automatic control system simulation models. The comparison results are shown in Table II.

Table II Comparison of control execution time (unit: s)

Number of The simulation model of automatic Simulation model of
Simulation model of 
INTERNATIONAL JOURNAL OF CIRCUITS, SYSTEMS AND SIGNAL PROCESSING

\begin{tabular}{cccc}
\hline experiments & control system constructed in this paper & $\begin{array}{c}\text { traditional automatic control } \\
\text { system } 1\end{array}$ & $\begin{array}{c}\text { traditional automatic control } \\
\text { system } 2\end{array}$ \\
\hline 5 & 0.86 & 2.56 & 3.25 \\
10 & 0.69 & 2.33 & 2.65 \\
15 & 1.01 & 2.85 & 3.32 \\
20 & 1.07 & 2.41 & 2.98 \\
25 & 0.96 & 2.69 & 3.02 \\
30 & 0.94 & 2.84 & 3.65 \\
35 & 0.97 & 2.65 & 3.12 \\
40 & 0.83 & 2.77 & 3.21 \\
45 & 1.14 & 2.91 & 3.67 \\
50 & 0.94 & 2.84 & 3.01 \\
Average & 0.94 & 2.69 & 3.19 \\
value & & &
\end{tabular}

By analyzing the data in Table II, it can be seen that the average control execution time of the simulation model of the automatic control system constructed in this paper is $0.94 \mathrm{~s}$, the average control execution time of the simulation model 1 of the traditional automatic control system is $2.69 \mathrm{~s}$, and the average control execution time of the simulation model 2 of the traditional automatic control system is $3.19 \mathrm{~s}$. The system in this paper is much lower than the traditional system. Therefore, it is proved that the simulation model of automatic control system constructed in this paper has better control execution efficiency and better practical application effect.

\section{DISCUSSION}

By comparing the execution time between the automatic control system simulation model proposed in this paper and the traditional automatic control system simulation model, it can be seen that the execution time of the vacuum nano coating automatic control system based on AVR MCU designed in this paper is far lower than the average control execution time of the simulation model 1 of the traditional automatic control system, and has more superior performance. The effect is better in practical application. This is because the automatic control system designed in this paper adopts an improved PID controller, which can effectively control the link of the comparative example, control the deviation of the link of the comparative example, reduce the steady-state error and optimize the performance of the control system.

However, the improved PID controller needs an accurate mathematical model, so it is necessary to improve the accurate calculation of the mathematical model. Under the condition of ensuring the normal operation of PID controller, the automatic control system designed in this paper still has the characteristics of high precision, fast time and good confidentiality, which can meet the practical application.

\section{CONCLUSION}

Based on the detailed analysis of the functional requirements and performance indexes of the system, the development of the automatic control system of vacuum nano coating based on AVR single chip microcomputer is completed through the overall scheme design, software and hardware development and joint debugging. When the simulation model and system parameters of the control system constructed in the environment change, and after adding interference signals, the designed system tends to remain stable within 15 seconds. It is proved that it has the characteristics of high precision, high speed, high reliability, good real-time performance, good stability, good confidentiality, and can meet the relevant needs well. In the future, it is necessary to verify the performance of the system in more and more complex scenarios, actively summarize and analyze the alternatives of the controller in the system according to the actual situation, and continuously optimize the performance of the system according to the relevant analysis results, so as to improve the comprehensive effect of vacuum nano coating and promote the further development of this field.

\section{References}

[1] S. Braden, H. Brandon and G. Adley, "Single chip lidar with discrete beam steering by digital micromirror device," Optics Express, vol. 25, no. 13, pp. 14732, 2017.

[2] M. Vilhelm and W. Fredrik, "Optical DNA mapping in nanofluidic channels: Principles and applications," Lab on A Chip, vol. 17, no. 4, pp. 579, 2017.

[3] P. Frédéric, C. Chitraleema and M. Muhammad, "Integration of single photon emitters in 2D layered materials with a silicon nitride photonic chip," Nature Communications, vol. 10, no. 1, pp. 1-7, 2019.

[4] J. Serafini, A. Hossain and R. B. James, "Photoconductive and electro-optic effects in (cd, mg) te single crystals measured in an experiment-on-chip configuration," Applied Physics Letters, vol. 111, no. 1, pp. 011108, 2017.

[5] V. Sundarapandian, "A new 3-d jerk chaotic system with two cubic nonlinearities and its adaptive backstepping control," Archives of Control Sciences, vol. 27, no. 3, pp. 409-439, 2017.

[6] P. Ohlemüller, F. Alobaid and A. Abad, "Development and validation of a $1 \mathrm{~d}$ process model with autothermal operation of a $1 \mathrm{mw}$ th chemical looping pilot plant," International Journal of Greenhouse Gas Control, vol. 73, pp. 29-41, 2018.

[7] B. D. Khuong, A. T. Khoa and P. Hannaford, "Spectrum modification of XUV radiation in the presence of a delayed 
weak control field," Journal of Modern Optics, vol. 66, no. 13, pp. 1-6, 2019.

[8] F. Adem, A. S. Idriz, R. Abdul and B. Simone, "Integration of auto-steering with adaptive cruise control for improved cornering behavior," IET Intelligent Transport Systems, vol. 11, no. 10, pp. 667-675, 2017.

[9] B. Manish, C. Shamim and A. Bilal, "Auto injection control for in-circuit frequency response measurement," IEEE Transactions on Industrial Electronics, vol. 99, pp. 1-1, 2017.

[10]Z. He, F. Zhou and X. Xia, "Interaction between oil price and investor sentiment: Nonlinear causality, time-varying influence, and asymmetric effect," Emerging Markets Finance and Trade, vol. 55, no. 12, pp. 2756-2773, 2019.

[11]G. Rohit and J. A. Phillip, "Closed-loop trailing-edge separation control system using empirical mode decomposition," AIAA Journal, vol. 56, no. 1, pp. 1-11, 2017.

[12]P. Sebastian and K. Stefan, "Koopman operator-based model reduction for switched-system control of PDEs," Automatica, vol. 106, pp. 184-191, 2019.

[13] A. Jeffrey, I. M. Davis and P. S. Jason, "Holographic projection system with programmable control of state of polarization focus plane and size of the reconstruction," Optical Engineering, vol. 58, no. 8, pp. 1, 2018.

[14] S. M. Bazhenov, S. A. Vakhonina and N. V. Tarasov, "Information and control system for automated weaving process monitoring," Automation and Remote Control, vol. 80, no. 3, pp. 576-583, 2019.

[15] F. Wen, L. Xu and G. Ouyang, "Retail investor attention and stock price crash risk: Evidence from China," International Review of Financial Analysis, vol. 65, pp. 101376, 2019.

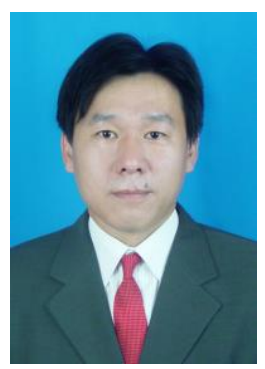

Jinwen Li, December 28, 1975, male, associate professor, bachelor degree, graduated from Chongqing University in 1997, majoring in Electromagnetic Measurement and Instrumentation; Master degree in engineering, graduated from Hefei University of Technology in 2016 in electrical engineering field; Now working in Chengde Petroleum College, teaching and scientific research in the direction of automation instruments, published 22 academic articles and participated in 6 scientific research projects.

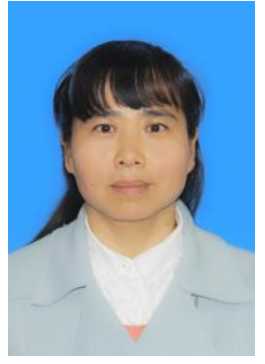

Jie Zhang, October 5, 1976, female, associate professor, bachelor degree, graduated from Northwest Institute of light industry in 2000, majoring in electrical technology; Master degree, graduated from North China Electric Power University in 2009, majoring in Power Electronics and Electrical Drive; Now working in Chengde Petroleum College, teaching and scientific research in the direction of electrical automation. published 12 academic articles and participated in 5 scientific research projects.

\section{Author Contribution}

Jinwen Li collected the samples, analysed the data. Jie Zhang conducted the experiments and analysed the results. All authors discussed the results and wrote the manuscript.

\section{Creative Commons Attribution License 4.0 (Attribution 4.0 International, CC BY 4.0)}

This article is published under the terms of the Creative Commons Attribution License 4.0 https://creativecommons.org/licenses/by/4.0/deed.en US 